\title{
Choosing wisely after publication of level I evidence in breast cancer radiotherapy
}

This article was published in the following Dove Press journal: Breast Cancer - Targets and Therapy

\author{
Joshua R Niska' \\ Sameer R Keole' \\ Barbara A Pockaj ${ }^{2}$ \\ Michele Y Halyard' \\ Samir H Patel' \\ Donald W Northfelt ${ }^{3}$ \\ Richard J Gray² \\ Nabil Wasif ${ }^{2}$ \\ Carlos E Vargas ${ }^{1}$ \\ William W Wong' \\ 'Department of Radiation Oncology, \\ ${ }^{2}$ Division of General Surgery, ${ }^{3}$ Division \\ of Hematology and Medical Oncology, \\ Mayo Clinic Hospital, Phoenix, AZ, \\ USA
}

Correspondence: Sameer R Keole Department of Radiation Oncology, Mayo Clinic Hospital, 5777 E Mayo Blvd, Phoenix, AZ 85054, USA

$\mathrm{Tel}+\mathrm{I} 480342$ I262

Fax +I 4803423972

Email keole.sameer@mayo.edu
Background: Recent trials in early-stage breast cancer support hypofractionated whole-breast radiotherapy (WBRT) as part of breast-conserving therapy (BCT). Evidence also suggests that radiotherapy (RT) omission may be reasonable for some patients over 70 years. Among radiationdelivery techniques, intensity-modulated RT (IMRT) is more expensive than 3-dimensional conformal RT (3DCRT). Based on this evidence, in 2013, the American Society for Radiation Oncology (ASTRO) recommended hypofractionated schedules for women aged $\geq 50$ years with early-stage breast cancer and avoiding routine use of IMRT for WBRT. To assess response to level I evidence and adherence to ASTRO recommendations, we evaluated the pattern of RT use for early-stage breast cancer at our National Comprehensive Cancer Network institution from 2006 to 2008 and 2011 to 2013 and compared the results with national trends.

Methods: Data from a prospective database were extracted to include patients treated with BCT, aged $\geq 50$ years, with histologic findings of invasive ductal carcinoma, stage T1-T2N0M0, estrogen receptor-positive, and HER2 normal. We retrospectively reviewed the medical records and estimated costs based on 2016 Hospital Outpatient Prospective Payment System (technical fees) and Medicare Physician Fee Schedule (professional fees).

Results: Among 55 cases from 2006 to 2008, treatment regimens were 11\% hypofractionated, $69 \%$ traditional schedule, and $20 \%$ RT omission ( $29 \%$ of patients were aged $>70$ years). Among 83 cases from 2011 to 2013 , treatment regimens were 54\% hypofractionated, $19 \%$ traditional schedule, and $27 \%$ RT omission ( $48 \%$ of patients were aged $>70$ years). 3DCRT was used for all WBRT treatments. Direct medical cost estimates were as follows: 15 fractions 3DCRT, $\$ 7,197.87 ; 15$ fractions IMRT, \$11,232.33; 25 fractions 3DCRT, \$9,731.39; and 25 fractions IMRT, $\$ 16,877.45$.

Conclusion: Despite apparent resistance to shorter radiation schedules in the United States, we demonstrate that rapid practice change in response to level I evidence is feasible. Wider adoption of evidence-based guidelines in early-stage breast cancer may substantially lower health care costs and improve convenience for patients without sacrificing oncologic outcomes.

Keywords: breast cancer, CALGB, choosing wisely, hypofractionation, omission, UK START

\section{Plain language summary}

Breast-conserving therapy allows many women with breast cancer to avoid mastectomy. After surgery, women have typically received at least 5 weeks of daily radiation to the whole breast. However, recent studies have shown that 3 weeks of daily radiation to the whole breast or even no radiation at all are options for some women. In addition, expensive radiation-delivery techniques may not be necessary for most whole-breast radiation. Whole breast radiation practice patterns in the US have been slow to change. For appropriately selected patients, we evaluated our 
practice patterns with regard to shorter radiation schedules, omission of whole-breast radiation, and use of different radiation-delivery techniques. We showed rapid practice change favoring shorter radiation schedules and omission of whole-breast radiation. Estimated cost savings by using shorter radiation schedules with appropriate radiation-delivery techniques may be substantial. Importantly, by adopting these practice changes, women with breast cancer are spared weeks of potentially unnecessary treatment.

\section{Introduction}

Adjuvant whole-breast radiotherapy (WBRT) plays an integral role in breast-conserving therapy (BCT) for women with early-stage breast cancer. In the landmark National Surgical Adjuvant Breast and Bowel Project ${ }^{1}$ and Milan ${ }^{2}$ trials, WBRT consisted of 50.0 Gy delivered in 25 daily fractions. However, patient inconvenience caused by 5 weeks of WBRT, increasing cost with each additional fraction, and limited resources led to interest in a shorter regimen. From a radiobiologic perspective, a shorter schedule delivering a higher daily dose of radiation over fewer treatment days (hypofractionated) can be equally effective. ${ }^{3}$ Concerns regarding long-term cosmetic results with a higher daily dose were allayed by 2 randomized trials in Canada and the United Kingdom. ${ }^{4,5}$ The hypofractionated schedules used in these trials thus seemed safe and would reduce the number of days required of patients while at the same time reducing resource utilization.

Three major randomized trials in Canada and the United Kingdom have now assessed traditional (50.0 Gy given in 25 fractions) versus hypofractionated WBRT for early-stage breast cancer. ${ }^{6-8}$ The Ontario Clinical Oncology Group (OCOG) compared traditional versus hypofractionated WBRT (42.5 Gy given in 16 fractions) after breast conservation surgery for early-stage breast cancer. At 10-year follow-up, Whelan et $\mathrm{al}^{6}$ reported no difference between the two fractionation schedules with regard to adverse effects, cosmetic outcome, local recurrence, or overall survival. The UK Standardisation of Breast Radiotherapy (START) Trialists' Group randomized women with early-stage breast cancer treated with primary surgery to either traditional WBRT or one of two hypofractionated schedules (41.6 Gy in 13 fractions or 39.0 Gy in 13 fractions). ${ }^{7}$ At 5-year follow-up, they found no difference in local recurrence and fewer adverse effects in the 39.0 Gy arm. The START Trialists' Group also performed START B, which randomized women with earlystage breast cancer to traditional versus hypofractionated WBRT (40.0 Gy in 15 fractions) after primary surgery. ${ }^{8}$ At 6-year follow-up, they found no difference in local recurrence. However, women in the hypofractionated arm had reduced late adverse effects, fewer distant metastases, greater disease-free survival, and greater overall survival. The benefits of the hypofractionated schedules in the START A and B trials were maintained at 10-year follow-up. ${ }^{9}$

In 2009, the National Institute for Health and Clinical Excellence in the United Kingdom recommended hypofractionated WBRT as standard of care for early-stage breast cancer. Most patients in the United Kingdom are now treated with hypofractionated schedules. ${ }^{10}$ Subsequently, the American Society for Radiation Oncology (ASTRO) issued an evidence-based guideline regarding fractionation for whole-breast irradiation. The ASTRO evidence-based guideline supports the use of hypofractionated WBRT for patients aged $\geq 50$ years, stage $\mathrm{pT} 1-\mathrm{T} 2 \mathrm{pN} 0 \mathrm{M} 0$, with no prior chemotherapy, as long as the hypofractionated radiation plan can achieve radiation dose heterogeneity of $\leq 7 \% .^{11}$

Although WBRT reduces the local recurrence rate for all breast cancers after breast-conserving surgery, the absolute benefit is quite small for elderly women. In the Cancer and Leukemia Group B (CALGB) 9343 study, women aged $\geq 70$ years with estrogen receptor-positive $(\mathrm{ER}+)$ early-stage breast cancer were randomized to lumpectomy plus tamoxifen with or without WBRT. ${ }^{12}$ At 10-year follow-up, CALGB 9343 showed a small improvement in local recurrence $(10 \%$ vs $2 \%$ ) and no difference in breast preservation, distant metastasis, and overall survival. ${ }^{12}$ National Comprehensive Center Network (NCCN) guidelines now reflect these data, offering radiotherapy (RT) omission as an option for elderly women with hormone receptor-positive, early-stage breast cancer. $^{13}$

In terms of treatment technique for delivery of WBRT, 3-dimensional conformal RT (3DCRT) or intensitymodulated RT (IMRT) are most common. The last cost comparison reported in the literature showed that a course of WBRT using IMRT was roughly twice the cost of 3DCRT. However, this information has now become outdated, as it was assessed using the 2003 Medicare fee schedule. ${ }^{14}$ One randomized trial reported clinically greater breast induration and change in breast appearance favoring IMRT versus 2-dimensional RT (2DRT), with no difference in quality of life. ${ }^{15}$ Randomized comparisons between IMRT and modern 3DCRT are lacking; however, one randomized trial compared IMRT with 2DRT, with computed tomography imaging used for planning in both arms. ${ }^{16}$ Reduced moist desquamation was associated with IMRT and smaller breast size. However, no difference in pain or quality of life was noted for IMRT versus 2DRT. ${ }^{16}$ Long-term follow-up showed no difference in chronic pain, quality of life, or cosmesis between IMRT 
and 2DRT. ${ }^{17}$ In the absence of clear clinical benefit, routine IMRT use for WBRT remains controversial.

On the basis of updated level I evidence, ASTRO released practice recommendations in 2013 as part of the national Choosing Wisely campaign. ${ }^{18}$ For breast cancer, clinicians are encouraged to 1) consider shorter treatment schedules for women aged $\geq 50$ years with early-stage breast cancer and 2) not routinely use IMRT to deliver WBRT.

To assess how practice patterns changed following the publication of level I evidence supporting hypofractionated WBRT, ${ }^{6-8}$ we evaluated the pattern of use of RT for women aged $\geq 50$ years with hormone receptor-positive, early-stage breast cancer at our NCCN member institution.

\section{Methods}

Two time periods were evaluated, 2006 through 2008 and 2011 through 2013, defined by date of lumpectomy. The time periods were selected to assess 3 years before and 3 years after publication of the START trials and the long-term follow-up of the OCOG trial.

Patients were identified from a prospective breast cancer database which along with this study, was approved by the Mayo Clinic Institutional Review Board, and written informed consent was obtained from all participants for the use of the data for research purposes. Inclusion criteria were completion of BCT at our institution, age $\geq 50$ years, histologic findings of invasive ductal carcinoma, stage T1-T2N0M0, ER+, and HER2 normal. These inclusion criteria were chosen to match the ASTRO recommended guidelines for shorter wholebreast radiation schedules. ${ }^{18}$ Patients receiving brachytherapy on clinical trial were excluded. We collected additional data from our institutional electronic medical record. Cases were categorized by RT modality (3DCRT, IMRT) and fractionation schedule (traditional $>23$ fractions vs hypofractionated $<17$ fractions, excluding boost).

Continuous variables were compared using the $t$ test. Categorical variables were compared using the chi-squared test. A $P$ value $<0.05$ was considered statistically significant. Cases were analyzed on an intent-to-treat basis.

Direct medical costs were estimated for 15- and 25-fraction WBRT using 3DCRT or IMRT, based on the Current Procedural Terminology, Fourth Edition, coding system. Payment rates are national average payments under the 2016 Hospital Outpatient Prospective Payment System (technical fees) and Medicare Physician Fee Schedule (professional fees). ${ }^{19,20}$

\section{Results}

From 2006 to 2008, 55 patients met the inclusion criteria (mean [SD] age, 72.1 [9.0] years; range $51-89$ years). WBRT was given on a traditional schedule for $69 \%$ of patients and was hypofractionated for $11 \%$. RT was omitted in $20 \%$ of all patients (mean [SD] age, 80.6 [6.3] years; range, 67-89 years). For patients aged $>70$ years, RT was omitted in $29 \%$.

From 2011 to 2013,83 patients met the inclusion criteria (mean [SD] age, 70.7 [8.9] years; range, 51-97 years). WBRT was given on a traditional schedule for $19 \%$ of patients and was hypofractionated for $54 \%$. RT was omitted for $27 \%$ of all patients (mean [SD] age, 78.5 [7.6] years; range, 61-97 years). For patients aged $>70$ years, RT was omitted in $48 \%$.

Between the two time periods, no significant differences were seen in patient age $(P=0.37)$, proportion of patients aged $>70$ years $(P=0.22)$, or disease stage $(P=0.95)$. The change in radiation therapy practice patterns between the two time periods was statistically significant $(P<0.001)$ (Table 1$)$. When only WBRT patients were considered, the hypofractionation rate increased from $14 \%$ in 2006-2008 to $74 \%$ in 2011-2013 $(P<0.001)$ (Figure 1). For patients aged $>70$ years, the RT omission rate increased from $29 \%$ in $2006-2008$ to $48 \%$ in 2011-2013; however, it did not reach statistical significance $(P=0.11)$ (Table 1; Figure 1).

The rationale for the treatment using a traditional rather than a hypofractionated schedule was documented in medical records in 11 of 16 traditional schedule cases from 2011 to 2013. In six cases, patient preference led to using the traditional schedule. In five cases, the treating physician recommended a traditional schedule because of a large breast separation. Of the five remaining cases in which the rationale for choosing a traditional schedule was not clear, one patient had breast implants and two patients were initially planned

Table I RT practice patterns for patients aged $\geq 50$ years with hormone receptor-positive, early-stage breast cancer at our NCCN-member institution

\begin{tabular}{|c|c|c|c|}
\hline \multirow[t]{2}{*}{ Characteristic } & \multicolumn{2}{|l|}{ Years } & \multirow[t]{2}{*}{$P$-value } \\
\hline & 2006-2008 & $2011-2013$ & \\
\hline Patients (N) & 55 & 83 & \\
\hline Age (SD), mean, years & $72.1(9.0)$ & 70.7 (8.9) & 0.37 \\
\hline \multicolumn{4}{|l|}{ Stage } \\
\hline TIa & 5 & 8 & 0.95 \\
\hline TIb & 21 & 29 & - \\
\hline TIc & 24 & 36 & - \\
\hline $\mathrm{T} 2$ & 5 & 10 & - \\
\hline \multicolumn{4}{|l|}{ RT practice patterns, N (\%) } \\
\hline Traditional WBRT & $38(69.1)$ & $16(19.3)$ & $<0.001$ \\
\hline Hypofractionated WBRT & $6(10.9)$ & $45(54.2)$ & - \\
\hline RT omission & II (20.0) & $22(26.5)$ & - \\
\hline Age $>70$ years, $N$ & 34 & 42 & 0.22 \\
\hline RT omission, N (\%) & $10(29.4)$ & $20(47.6)$ & 0.11 \\
\hline
\end{tabular}


for hypofractionation but were ultimately treated with a traditional schedule.

All WBRT was delivered using 3DCRT. No cases used an IMRT technique. WBRT was delivered in 2.66 or $2.67 \mathrm{~Gy} /$ fraction for the hypofractionated regimen and $2 \mathrm{~Gy} /$ fraction for the traditional regimen.

Direct medical cost estimates were as follows: 15 fractions of 3DCRT, \$7,197.87; 15 fractions of IMRT, \$11,232.33; 25 fractions of 3DCRT, \$9,731.39; and 25 fractions of IMRT, $\$ 16,877.45$ (Table 2).

\section{Discussion}

Among patients with early-stage breast cancer who had WBRT at our NCCN member institution, the hypofractionation rate increased from $14 \%$ in $2006-2008$ to $74 \%$ in $2011-2013$ (Figure 1). These hypofractionation rates compare favorably to national rate trends, including the subset of academic institutions. An analysis based on the Surveillance, Epidemiology, and End Results (SEER)-Medicare-linked database showed hypofractionation rates of $10 \%$ in 2008 and $14 \%$ in 2009-2010 for early-stage breast cancer. ${ }^{21}$ Retrospective data from the National Cancer Database demonstrated that for patients deemed eligible for hypofractionation, national hypofractionation rates increased from $10 \%$ in 2008 to $23 \%$ in 2011 , with increasing rates of hypofractionation in academic institutions from $\sim 16 \%$ in 2008 to $\sim 32 \%$ in $2011 .^{22}$ More recently, a report from the Michigan Radiation Oncology Quality Consortium showed that $31 \%$ of patients

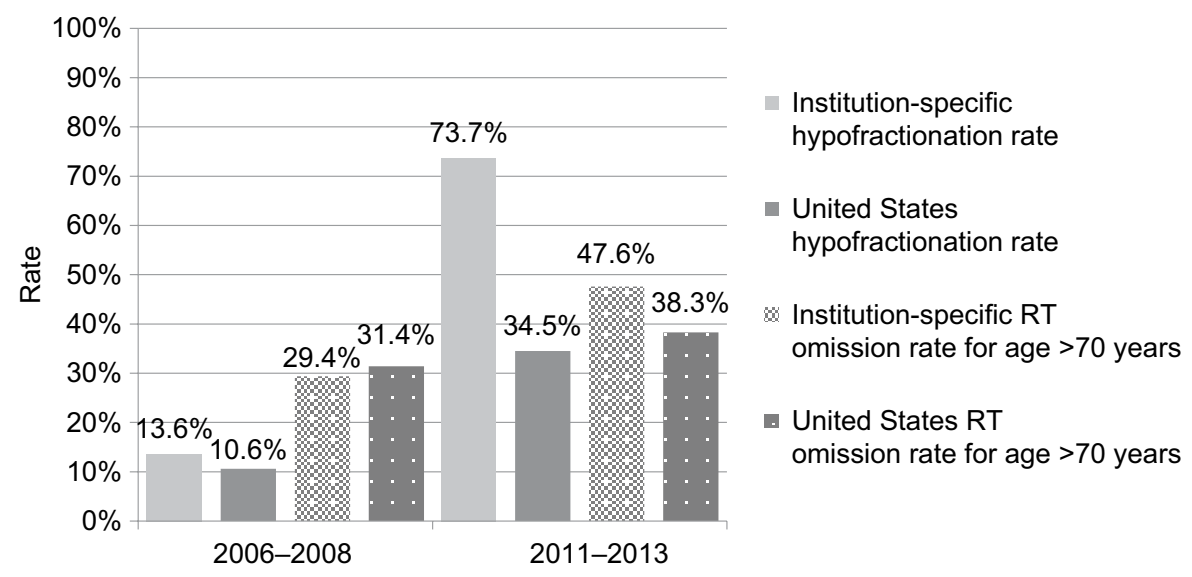

Figure I Rates of hypofractionation and RT omission for the years 2006-2008 and 20I I-20I3. WBRT hypofractionation rates are for patients aged $\geq 50$ years with hormone receptor-positive, early-stage breast cancer. RT omission rates are for patients aged $>70$ years. Rates at our NCCN-member institution are compared to national rates per Bekelman et $\mathrm{a}^{24}$ and Palta et al. ${ }^{25} P<0.00 \mathrm{I}$ for statistical difference between the 2006-2008 and $201 \mathrm{I}-20 \mathrm{I} 3$ institution-specific hypofractionation rates. $P=0.1 \mathrm{I}$ for statistical difference between the 2006-2008 and 20II-20I3 institution-specific RT omission rates.

Abbreviations: NCCN, National Comprehensive Center Network; RT, radiotherapy; WBRT, whole-breast radiotherapy.

Table 2 Direct medical cost for whole-breast radiotherapy

\begin{tabular}{|c|c|c|c|c|c|c|c|c|}
\hline Code & Description & Technical & Professional & Total & 3DCRT & IMRT & 3DCRT & IMRT \\
\hline & & cost $(\$)$ & cost (\$) & cost (\$) & 15 fractions & 15 fractions & 25 fractions & 25 fractions \\
\hline 77263 & Clinical treatment planning, complex & - & 167.56 & 167.56 & 1 & 1 & 1 & 1 \\
\hline 77280 & Simulation, simple & 166.65 & 36.52 & 203.17 & I & - & 1 & - \\
\hline 77290 & Simulation, complex & 291.77 & 81.99 & 373.76 & 1 & - & 1 & - \\
\hline 77293 & Respiratory motion management & - & 104.91 & 104.91 & 0.5 & - & 0.5 & - \\
\hline 77295 & 3DCRT treatment plan & $\mathrm{I}, 026.8 \mathrm{I}$ & 224.13 & $\mathrm{I}, 250.94$ & $\mathrm{I}$ & - & 1 & - \\
\hline 77300 & Dosimetry calculation & 107.40 & 32.58 & 139.98 & 2 & 2 & 2 & 2 \\
\hline 77301 & IMRT treatment plan & $\mathrm{I}, 026.8 \mathrm{I}$ & 417.84 & $\mathrm{I}, 444.65$ & - & I & - & I \\
\hline 77321 & Electron boost plan & 291.77 & 49.77 & 341.54 & - & - & - & - \\
\hline 77334 & Treatment devices & 291.77 & 64.81 & 356.58 & 3 & 1 & 3 & 1 \\
\hline 77336 & Weekly physics & 107.40 & - & 107.40 & 3 & 3 & 5 & 5 \\
\hline 77338 & Design MLC for IMRT & 291.77 & 224.13 & 515.90 & - & I & - & I \\
\hline 77385 & IMRT treatment delivery, simple & 505.51 & - & 505.51 & - & 15 & - & 25 \\
\hline 77412 & 3DCRT/electron treatment delivery & 194.35 & - & 194.35 & 15 & - & 25 & - \\
\hline 77417 & Weekly port films & NC & - & - & 3 & - & 5 & - \\
\hline 77427 & Weekly RT management & - & $|87.6|$ & $|87.6|$ & 3 & 3 & 5 & 5 \\
\hline Direct & medical cost (\$) & & & & $7,197.87$ & $\mathrm{II}, 232.33$ & $9,731.39$ & $16,877.45$ \\
\hline
\end{tabular}

Abbreviations: -, not applicable; 3DCRT, 3-dimensional conformal radiotherapy; IMRT, intensity-modulated radiotherapy; MLC, multileaf collimator; NC, no charge; RT, radiotherapy. 
with early-stage breast cancer registered from October 2011 to December 2013 received hypofractionated WBRT. ${ }^{23}$ A retrospective analysis using administrative claims data from commercial health care plans covering $7 \%$ of the adult female population in the USA showed that the rates of hypofractionated WBRT for women aged $\geq 50$ years with early-stage breast cancer were $11 \%$ in 2008 and $35 \%$ in $2013 .{ }^{24}$ While these comparisons show a trend toward increased hypofractionation nationwide, our institution seems to have adopted the shorter treatment schedule to a far greater extent.

RT omission for patients aged $>70$ years with early-stage breast cancer increased by nearly $20 \%$ between the two periods we evaluated at our institution (29\%-48\%, Figure 1$)$. According to a SEER database analysis, national rates of RT omission for this group were $31 \%$ between 2000 and 2004 and 38\% between 2005 and $2009 .{ }^{25}$ As of 2011-2013, the rate of RT omission for women aged $>70$ years at our institution exceeds the published national rates. Based on CALGB $9343,{ }^{12}$ current NCCN guidelines ${ }^{13}$ use age 70 years as a cutoff for the selected option of RT omission. However, multiple additional trials are now evaluating RT omission for younger patients who are still at low risk of recurrence. PRIME II has published 5-year data on RT omission for patients aged $\geq 65$ years. ${ }^{26}$ Ongoing studies include the IDEA Study $^{27}$ (postmenopausal patients), the PRECISION Trial ${ }^{28}$ (patients aged $\geq 50$ years), and the EXPERT Trial ${ }^{29}$ (patients aged $\geq 50$ years). If long-term results from these trials are favorable, RT omission may become an option for even younger patients.

Of the 16 patients who received the traditional schedule WBRT from 2011 to 2013, six patients preferred the longer schedule and five had a large breast separation, which has been a perceived contraindication to hypofractionated WBRT. ${ }^{6}$ Thus, only five of 88 patients were treated with the longer traditional radiation schedule without a clearly documented rationale for not using the shorter hypofractionated schedule. Although the OCOG trial excluded women with a maximum width of breast tissue of over $25 \mathrm{~cm}$, the START trials had no breast separation limitations. ${ }^{6-8}$ Other studies have shown larger body habitus (proxy for large breast separation) to be associated with lack of hypofractionation. ${ }^{21,23}$ IMRT may be an appropriate modality to minimize adverse effects for patients with large breast separation who are undergoing hypofractionation, thus broadening the applicability of the hypofractionated schedule. ${ }^{30}$

Factors associated with higher hypofractionation rates include both patient and institutional characteristics, such as living $\geq 50$ miles from the cancer reporting facility, ${ }^{22}$ treatment at an academic center, ${ }^{22}$ higher median income, ${ }^{22,24}$ older age,,$^{21,23,24}$ higher education level, living in a western SEER region, and more comorbidity. ${ }^{21}$ Bekelman et a ${ }^{24}$ also found hypofractionation more likely to occur in an outpatient hospital setting versus a freestanding facility. Our institution is a tertiary academic center in the western USA that spans both outpatient hospital and freestanding facilities. Although we did not investigate these patient demographic characteristics, it is possible that the patient population in our study is unbalanced with regard to the abovementioned variables, resulting in selection bias.

In contrast to the slower national uptake of hypofractionated WBRT and the selected option of RT omission, IMRT has shown rapid acceptance despite limited supportive evidence. Among patients with node-negative breast cancer, IMRT use in breast conservation increased from $9 \%$ in 2004 to $23 \%$ in 2009 to $2010 .{ }^{21}$ IMRT was not used at our institution for patients aged $\geq 50$ years with hormone receptor-positive, early-stage breast cancer. Some providers argue that IMRT can deliver a more homogeneous radiation dose and thus reduce toxicity. However, our practice is to use field-in-field 3DCRT technique, which achieves dose homogeneity comparable to inverse-planned IMRT. ${ }^{31}$ Although hypofractionated regimens and RT omission reduce cost, IMRT is more costly than 3DCRT. ${ }^{14}$ At current reimbursement levels, IMRT is associated with an estimated added cost of $\$ 4,034.46$ and $\$ 7,146.06$ for 15- and 25-fraction WBRT courses, respectively (Table 2). Whether providers are making a conscious effort or not, it would stand to reason that financially advantageous practices, such as the use of IMRT, would be used more commonly than their cheaper counterparts. This type of financial incentive has been previously demonstrated for IMRT use among self-referring urologists ${ }^{32}$ and may similarly impact national IMRT utilization, as well as lack of hypofractionation and RT omission in early-stage breast cancer.

Potential cost savings may be substantial if providers follow evidence-based practice guidelines and expert recommendations for selected patients with early-stage breast cancer. According to the ASTRO evidence-based guidelines, each year, about 20,000 women who have WBRT are eligible for hypofractionation. About 2,000 of these patients ultimately receive IMRT. About 4,000 are aged $>70$ years and eligible for RT omission. ${ }^{24}$ By our own assessment (Table 2 ), the direct medical cost of a single course of WBRT using 15 fractions 3DCRT is less than half the cost of 25 fractions IMRT, a savings of nearly $\$ 10,000$ in direct medical costs per patient by selecting hypofractionated 3DCRT over traditional course IMRT. RT omission removes the cost of RT entirely, 
a savings of about $\$ 7,000-\$ 17,000$ per patient. Practice patterns with regard to WBRT for early-stage breast cancer affect thousands of patients per year, who could each save thousands of dollars in direct medical costs if hypofractionation or RT omission is chosen. Given these data, choosing hypofractionated WBRT when appropriate, omitting RT for selected elderly women, and avoiding unnecessary IMRT could ultimately account for millions of dollars in direct medical cost savings to the US healthcare system - all while adopting evidence-based practices.

\section{Limitations}

Limitations of the current study include the small sample size and its retrospective nature. Our institution is an NCCN member that establishes institutional guidelines across three national sites and may, therefore, stand as an outlier among national RT providers. There are additional scenarios beyond the scope of this paper, including longer treatment schedules using $1.8 \mathrm{~Gy} /$ fraction or lumpectomy cavity boost, or both, which add time, fractions, and expenses. Our institution uses the Radiation Therapy Oncology Group 1005 indications for lumpectomy cavity boost as a guideline, which spares many patients' additional fractions. Additional studies have evaluated even shorter regimens, delivering five fractions once weekly ${ }^{33}$ or five fractions in 1 week. ${ }^{34}$ We understand that practice patterns may vary nationally and have only presented the direct medical costs associated with the four WBRT regimens most applicable to our discussion.

\section{Conclusion}

Overall, the data presented show that it is possible for RT practice patterns to rapidly change in response to new level I evidence. We demonstrate evidence-based practice and adherence to national expert recommendations for the population of women aged $\geq 50$ years with hormone receptor-positive, early-stage breast cancer. Millions of potential savings in the health care system could be achieved by practicing evidence-based medicine and following national guidelines for RT in early-stage breast cancer. More importantly, shorter treatment schedules and omission of WBRT in selected patients do not compromise oncologic outcomes and have the immeasurable benefit of avoiding weeks of potentially unnecessary RT to patients.

\section{Acknowledgments}

We are deeply grateful to Sally Eggleston, MBA, BSRT(T) of Revenue Cycle, Inc (Austin, Texas, USA) for her assistance with our cost assessment. Portions of this manuscript have been published in abstract form: Int J Radiat Oncol Biol Phys. 2015;93(3 Suppl):E12.

\section{Disclosure}

The authors report no conflicts of interest in this work.

\section{References}

1. Fisher B, Anderson S, Bryant J, et al. Twenty-year follow-up of a randomized trial comparing total mastectomy, lumpectomy, and lumpectomy plus irradiation for the treatment of invasive breast cancer. $N E n g l$ J Med. 2002;347(16):1233-1241.

2. Veronesi U, Luini A, Del Vecchio M, et al. Radiotherapy after breastpreserving surgery in women with localized cancer of the breast. $N$ Engl J Med. 1993;328(22):1587-1591.

3. Fowler JF. The linear-quadratic formula and progress in fractionated radiotherapy. Br J Radiol. 1989;62(740):679-694.

4. Olivotto IA, Weir LM, Kim-Sing C, et al. Late cosmetic results of short fractionation for breast conservation. Radiother Oncol. 1996;41(1):7-13.

5. Yarnold J, Ashton A, Bliss J, et al. Fractionation sensitivity and dose response of late adverse effects in the breast after radiotherapy for early breast cancer: long-term results of a randomised trial. Radiother Oncol. 2005;75(1):9-17.

6. Whelan TJ, Pignol JP, Levine MN, et al. Long-term results of hypofractionated radiation therapy for breast cancer. $N$ Engl J Med. 2010;362(6): 513-520.

7. Group ST, Bentzen SM, Agrawal RK, et al. The UK Standardisation of Breast Radiotherapy (START) Trial A of radiotherapy hypofractionation for treatment of early breast cancer: a randomised trial. Lancet Oncol. 2008;9(4):331-341.

8. Group ST, Bentzen SM, Agrawal RK, et al. The UK Standardisation of Breast Radiotherapy (START) Trial B of radiotherapy hypofractionation for treatment of early breast cancer: a randomised trial. Lancet. 2008;371(9618):1098-1107.

9. Haviland JS, Owen JR, Dewar JA, et al. The UK Standardisation of Breast Radiotherapy (START) trials of radiotherapy hypofractionation for treatment of early breast cancer: 10-year follow-up results of two randomised controlled trials. Lancet Oncol. 2013;14(11):1086-1094.

10. Harnett A. Fewer fractions of adjuvant external beam radiotherapy for early breast cancer are safe and effective and can now be the standard of care: why the UK's NICE accepts fewer fractions as the standard of care for adjuvant radiotherapy in early breast cancer. Breast. 2010;19(3):159-162.

11. Smith BD, Bentzen SM, Correa CR, et al. Fractionation for whole breast irradiation: an American Society for Radiation Oncology (ASTRO) evidence-based guideline. Int J Radiat Oncol Biol Phys. 2011;81(1):59-68.

12. Hughes KS, Schnaper LA, Bellon JR, et al. Lumpectomy plus tamoxifen with or without irradiation in women age 70 years or older with early breast cancer: long-term follow-up of CALGB 9343. J Clin Oncol. 2013;31(19):2382-2387.

13. National Comprehensive Cancer Network Clinical Practice Guidelines in Oncology. Breast cancer. Available from: https://www.nccn.org/ professionals/physician_gls/pdf/breast.pdf. Accessed December 14, 2017.

14. Suh WW, Pierce LJ, Vicini FA, Hayman JA. A cost comparison analysis of partial versus whole-breast irradiation after breast-conserving surgery for early-stage breast cancer. Int J Radiat Oncol Biol Phys. 2005;62(3):790-796.

15. Donovan E, Bleakley N, Denholm E, et al. Randomised trial of standard $2 \mathrm{D}$ radiotherapy (RT) versus intensity modulated radiotherapy (IMRT) in patients prescribed breast radiotherapy. Radiother Oncol. 2007;82(3):254-264.

16. Pignol JP, Olivotto I, Rakovitch E, et al. A multicenter randomized trial of breast intensity-modulated radiation therapy to reduce acute radiation dermatitis. J Clin Oncol. 2008;26(13):2085-2092.

17. Pignol JP, Truong P, Rakovitch E, Sattler MG, Whelan TJ, Olivotto IA. Ten years results of the Canadian breast intensity modulated radiation therapy (IMRT) randomized controlled trial. Radiother Oncol. 2016;121(3):414-419. 
18. American Society for Radiation Oncology. ASTRO releases list of five radiation oncology treatments to question as part of national Choosing Wisely campaign. 2013. Available from: http://www.choosingwisely. org/astro-releases-list-of-five-radiation-oncology-treatments-toquestion-as-part-of-national-choosing-wisely-campaign. Accessed December 14, 2017.

19. Centers for Medicare and Medicaid Services. Physician fee schedule. Available from: https://www.cms.gov/apps/physician-fee-schedule/. Accessed August 29, 2016.

20. Centers for Medicare and Medicaid Services. Hospital outpatient prospective payment system. Available from: https://www.cms.gov/ Medicare/Medicare-Fee-for-Service-Payment/HospitalOutpatientPPS/. Accessed August 29, 2016.

21. Jagsi R, Falchook AD, Hendrix LH, Curry H, Chen RC. Adoption of hypofractionated radiation therapy for breast cancer after publication of randomized trials. Int J Radiat Oncol Biol Phys. 2014;90(5):1001-1009.

22. Wang EH, Mougalian SS, Soulos PR, et al. Adoption of hypofractionated whole-breast irradiation for early-stage breast cancer: a National Cancer Data Base analysis. Int J Radiat Oncol Biol Phys. 2014;90(5):993-1000.

23. Jagsi R, Griffith KA, Heimburger D, et al. Choosing wisely? Patterns and correlates of the use of hypofractionated whole-breast radiation therapy in the state of Michigan. Int J Radiat Oncol Biol Phys. 2014;90(5):1010-1016.

24. Bekelman JE, Sylwestrzak G, Barron J, et al. Uptake and costs of hypofractionated vs conventional whole breast irradiation after breast conserving surgery in the United States, 2008-2013. JAMA. 2014;312(23):2542-2550.

25. Palta M, Palta P, Bhavsar NA, Horton JK, Blitzblau RC. The use of adjuvant radiotherapy in elderly patients with early-stage breast cancer: changes in practice patterns after publication of Cancer and Leukemia Group B 9343. Cancer. 2015;121(2):188-193.

26. Kunkler IH, Williams LJ, Jack WJ, Cameron DA, Dixon JM; PRIME II Investigators. Breast-conserving surgery with or without irradiation in women aged 65 years or older with early breast cancer (PRIME II) a randomised controlled trial. Lancet Oncol. 2015;16(3):266-273.
27. University of Michigan Cancer Center. The IDEA Study (Individualized Decisions for Endocrine Therapy Alone). Available from: https:// clinicaltrials.gov/ct2/show/NCT02400190. Accessed December 14, 2017.

28. Dana-Farber Cancer Institute. The PRECISION Trial (Profiling Early Breast Cancer for Radiotherapy Omission): A Phase II Study of BreastConserving Surgery Without Adjuvant Radiotherapy for Favorable-Risk Breast Cancer. Available from: https://clinicaltrials.gov/ct2/show/ NCT02653755. Accessed December 14, 2017.

29. Breast Cancer Trials, Australia and New Zealand. EXamining PErsonalised Radiation Therapy for Low-risk Early Breast Cancer (EXPERT). Available from: https:/clinicaltrials.gov/ct2/show/NCT02889874. Accessed December 14, 2017.

30. Hannan R, Thompson RF, Chen Y, et al. Hypofractionated whole-breast radiation therapy: does breast size matter? Int J Radiat Oncol Biol Phys. 2012;84(4):894-901.

31. Al-Rahbi ZS, Al Mandhari Z, Ravichandran R, et al. Dosimetric comparison of intensity modulated radiotherapy isocentric field plans and field in field (FIF) forward plans in the treatment of breast cancer. J Med Phys. 2013;38(1):22-29.

32. Mitchell JM. Urologists' use of intensity-modulated radiation therapy for prostate cancer. $N$ Engl J Med. 2013;369(17):1629-1637.

33. Dragun AE, Quillo AR, Riley EC, et al. A phase 2 trial of once-weekly hypofractionated breast irradiation: first report of acute toxicity, feasibility, and patient satisfaction. Int J Radiat Oncol Biol Phys. 2013;85(3):e123-e128.

34. Brunt AM, Wheatley D, Yarnold J, et al; FAST-Forward Trial Management Group. Acute skin toxicity associated with a 1-week schedule of whole breast radiotherapy compared with a standard 3-week regimen delivered in the UK FAST-Forward Trial. Radiother Oncol. 2016;120(1): 114-118.
Breast Cancer - Targets and Therapy

\section{Publish your work in this journal}

Breast Cancer - Targets and Therapy is an international, peerreviewed open access journal focusing on breast cancer research, identification of therapeutic targets and the optimal use of preventative and integrated treatment interventions to achieve improved outcomes, enhanced survival and quality of life for the cancer patient.

\section{Dovepress}

The manuscript management system is completely online and includes a very quick and fair peer-review system, which is all easy to use. Visit http://www.dovepress.com/testimonials.php to read real quotes from published authors. 